\title{
PERSPECTIVES ON INFORMATION-BASED COMPLEXITY
}

\author{
J. F. TRAUB AND H. WOŹNIAKOWSKI
}

\section{INTRODUCTION}

Computational complexity studies the intrinsic difficulty of mathematically posed problems and seeks optimal means for their solutions. This is a rich and diverse field; for the purpose of this paper we present a greatly simplified picture.

Computational complexity may be divided into two branches, discrete and continuous. Discrete computational complexity studies problems such as graph theoretic, routing, and discrete optimization; see, for example, Garey and Johnson [79]. Continuous computational complexity studies problems such as ordinary and partial differential equations, multivariate integration, matrix multiplication, and systems of polynomial equations. Discrete computational complexity often uses the Turing machine model whereas continuous computational complexity tends to use the real number model.

Continuous computational complexity may again be split into two branches. The first deals with problems for which the information is complete. Problems where the information may be complete are those for which the input is specified by a finite number of parameters. Examples include linear algebraic systems, matrix multiplication, and systems of polynomial equations. Recently, Blum, Shub and Smale [89] obtained the first NP-completeness result over the reals for a problem with complete information.

The other branch of continuous computational complexity is informationbased complexity, which is denoted for brevity as IBC. Typically, IBC studies infinite-dimensional problems. These are problems where either the input or the output are elements of infinite-dimensional spaces. Since digital computers can handle only finite sets of numbers, infinite-dimensional objects such as functions on the reals must be replaced by finite sets of numbers. Thus, complete information is not available about such objects. Only partial information is available when solving an infinite-dimensional problem on a digital computer. Typically, information is contaminated with errors such as round-off error, measurement error, and human error. Thus, the available information is partial and/or contaminated.

We want to emphasize this point for it is central to IBC. Since only partial and/or contaminated information is available, we can solve the original problem only approximately. The goal of IBC is to compute such an approximation as inexpensively as possible.

In Figure 1 (see p. 30) we schematize the structure of computational complexity described above.

Received by the editors April, 1991.

1991 Mathematics Subject Classification. Primary 68Q25.

This research was supported in part by the National Science Foundation. 


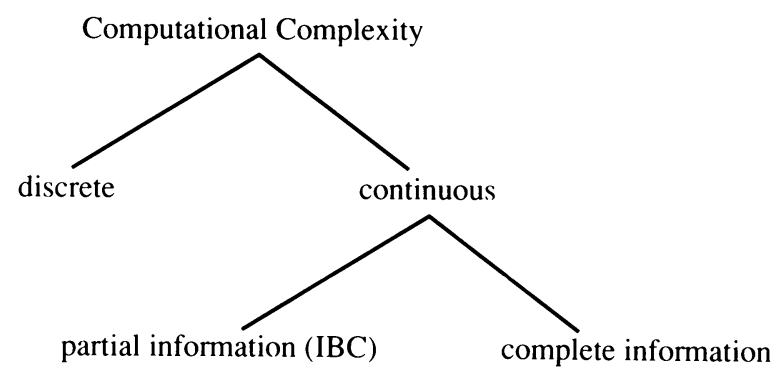

FIGURE 1

Research in the spirit of IBC was initiated in the Soviet Union by Kolmogorov in the late 1940s. Nikolskij [50], then a graduate student of Kolmogorov, studied optimal quadrature. This line of research was greatly advanced by Bakhvalov; see, e.g., Bakhvalov [59, 64, 71]. In the United States research in the spirit of IBC was initiated by Sard [49] and Kiefer [53]. Kiefer reported the results of his 1948 MIT Master's Thesis that Fibonacci sampling is optimal when approximating the maximum of a unimodal function. Sard studied optimal quadrature. Golomb and Weinberger [59] studied optimal approximation of linear functionals. Schoenberg [64] realized the close connection between splines and algorithms optimal in the sense of Sard.

IBC is formulated as an abstract theory and it has applications in numerous areas. The reader may consult TWW $[88]^{1}$ for some of the applications. IBC has benefitted from research in many fields. Influential have been questions, concepts, and results from complexity theory, algorithmic analysis, applied mathematics, numerical analysis, statistics, and the theory of approximation (particularly the work on $n$-widths and splines).

In this paper we discuss, in particular, IBC research for two problems of numerical analysis. We first contrast IBC and numerical analysis, limiting ourselves to just one characteristic of each.

IBC is a branch of computational complexity, and optimal (or almost optimal) information and algorithms are obtained from the theory. In numerical analysis, particular classes of algorithms are carefully analyzed to see if they satisfy certain criteria such as convergence, error bounds, efficiency, and stability.

Numerical analysis and IBC have different views on the problems which lie in their common domain. The authors of this paper have worked in both numerical analysis and IBC, and believe the viewpoints are not right or wrong, just different.

On the other hand, in many research groups around the world, people work on both numerical analysis and IBC, and do not draw a sharp distinction between the two. They believe IBC can serve as part of the theoretical foundation of numerical analysis.

We believe there might be some profit in discussing the views of numerical analysis and IBC. Unfortunately Parlett $[92]^{2}$ does not serve this purpose since, as we shall show, this paper ignores relevant literature and is mistaken on issues of complexity theory.

\footnotetext{
${ }^{1}$ When one of us is a coauthor, the citation will be made using only initials.

${ }^{2}$ Citation to this paper will be made using only an initial.
} 
For example, $\mathbf{P}$ [92] contains a central misconception about IBC which immediately invalidates large portions of the paper. $P$ [92] assumes that the information is specified (or fixed). Indeed, the first "high level criticism" is that IBC "is not complexity theory" (see P [92, 2.A]), since "specified information" is used.

But it is the very essence of IBC that both the information and the algorithms are varied. Indeed, one of the central problems of IBC is the optimal choice of information. Significant portions of three monographs, TW [80] and TWW [83, 88], all of which are cited in P [92], are devoted to this issue. We return to this issue in $\S 3$ after notation has been established.

In $\mathbf{P}$ [92], the author limits himself to "matrix computations, which is the area we understand best." We do not object to discussing matrix computations, although they constitute a small fraction and are atypical of IBC. For example, in the recent monograph TWW [88], some ten pages, just $2 \%$, are devoted to matrix computations. Matrix computations are atypical since complete information can be obtained at finite cost. However, even in this particular area, P [92] ignores relevant literature and does not exhibit a grasp of the complexity issues. Since the discussion will, of necessity, assume some rather technical details concerning matrix computations, we will defer it to $\S \S 5$ and 6 .

We stress that we are not questioning the importance of matrix computations. On the contrary, they play a central role in scientific computation. Furthermore, we believe there are some nice results and deep open questions regarding matrix computations in IBC.

But the real issue is, after all, IBC in its entirety. P [92] is merely using the two papers TW [84] and Kuczyński [86] on matrix computations to criticize all of IBC. We therefore respond to general criticisms in $\S \S 3$ and 4.

To make this paper self-contained we briefly summarize the basic concepts of IBC in $\S 2$. Section 7 deals with possible refinements of IBC. A summary of our rebuttal to criticisms in $P$ [92] is presented in $\S 8$.

\section{OUTLINE OF IBC}

In this section we introduce the basic concepts of IBC and define the notation which will be used for the remainder of this paper. We illustrate the concepts with the example of multivariate integration, a typical application of IBC. A more detailed account may be found in TWW [88]. Expository material may be found in W [85], PT [87], PW [87], and TW [91]. Let

$$
S: F \rightarrow G,
$$

where $F$ is a subset of a linear space and $G$ is a normed linear space. We wish to compute an approximation to $S(f)$ for all $f$ from $F$.

Typically, $f$ is an element from an infinite-dimensional space and it cannot be represented on a digital computer. We therefore assume that only partial information $^{3}$ about $f$ is available. We gather this partial information about $f$ by computing information operations $L(f)$, where $L \in \Lambda$. Here the class $\Lambda$ denotes a collection of information operations that may be computed. We illustrate these concepts by an example.

\footnotetext{
${ }^{3}$ For simplicity, we will not consider contaminated information in this paper.
} 
Example: Multivariate integration. Let $F$ be a unit ball of the Sobolev class $W_{p}^{r, d}$ of real functions defined on the $d$-dimensional cube $D=[0,1]^{d}$ whose $r$ th distributional derivatives exist and are bounded in $L_{p}$ norm. Let $G=\mathbb{R}$ and

$$
S(f)=\int_{D} f(t) d t
$$

Assume $p r>d$. To approximate $S(f)$, we assume we can compute only function values. That is, the class $\Lambda$ is a collection of $L: F \rightarrow \mathbb{R}$, such that for some $x$ from $D, L(f)=f(x), \forall f \in F$.

For each $f \in F$, we compute a number of information operations from the class $\Lambda$. Let

$$
N(f)=\left[L_{1}(f), L_{2}(f), \ldots, L_{n}(f)\right], \quad L_{i} \in \Lambda,
$$

be the computed information about $f$. We stress that the $L_{i}$ as well as the number $n$ can be chosen adaptively. That is, the choice of $L_{i}$ may depend on the already computed $L_{1}(f), L_{2}(f), \ldots, L_{i-1}(f)$. The number $n$ may also depend on the computed $L_{i}(f)$. (This permits arbitrary termination criteria.)

$N(f)$ is called the information about $f$, and $N$ the information operator. In general, $N$ is many-to-one, and that is why it is impossible to recover the element $f$, knowing $y=N(f)$ for $f \in F$. For this reason, the information $N$ is called partial.

Having computed $N(f)$, we approximate $S(f)$ by an element $U(f)=$ $\phi(N(f))$, where $\phi: N(F) \rightarrow G$. A mapping $\phi$ is called an algorithm.

The definition of error of the approximation $U$ depends on the setting. We restrict ourselves here to only two settings. In the worst case setting

$$
e(U)=\sup _{f \in F}\|S(f)-U(f)\|,
$$

and in the average case setting, given a probability measure $\mu$ on $F$,

$$
e(U)=\left(\int_{F}\|S(f)-U(f)\|^{2} \mu(d f)\right)^{1 / 2} .
$$

Example (continued). The information is given by

$$
N(f)=\left[f\left(x_{1}\right), f\left(x_{2}\right), \ldots, f\left(x_{n}\right)\right]
$$

with the points $x_{i}$ and the number $n$ adaptively chosen. An example of an algorithm is a linear algorithm given by $U(f)=\phi(N(f))=\sum_{i=1}^{n} a_{i} f\left(x_{i}\right)$ for some numbers $a_{i}$.

In the worst case setting, the error is defined as the maximal distance $\mid S(f)-$ $U(f) \mid$ in the set $F$. In the average case setting, the error is the $L_{2}$ mean of $|S(f)-U(f)|$ with respect to the probability measure $\mu$. The measure $\mu$ is sometimes taken as a truncated Gaussian measure.

To define the computational complexity we need a model of computation. It is defined by two assumptions:

(1) We are charged for each information operation. That is, for every $L \in$ $\Lambda$ and for every $f \in F$, the computation of $L(f)$ costs $c$, where $c$ is positive and fixed, independent of $L$ and $f$. 
(2) Let $\Omega$ denote the set of permissible combinatory operations including the addition of two elements in $G$, multiplication by a scalar in $G$, arithmetic operations, comparison of real numbers, and evaluations of certain elementary functions. We assume that each combinatory operation is performed exactly with unit cost.

In particular, this means that we use the real number model, where we can perform operations on real numbers exactly and at unit cost. Modulo roundoffs and the very important concept of numerical stability, this corresponds to floating point arithmetic widely used for solving scientific computational problems.

We now define the cost of the approximations. Let $\operatorname{cost}(N, f)$ denote the cost of computing the information $N(f)$. Note that $\operatorname{cost}(N, f) \geq c n$, and the inequality may occur since adaptive selection of $L_{i}$ and $n$ may require some combinatory operations.

Knowing $y=N(f)$, we compute $U(f)=\phi(y)$ by combining the information $L_{i}(f)$. Let $\operatorname{cost}(\phi, y)$ denote the number of combinatory operations from $\Omega$ needed to compute $\phi(y)$. We stress that $\operatorname{cost}(N, f)$ or $\operatorname{cost}(\phi, y)$ may be equal to infinity if $N(f)$ or $\phi(y)$ use an operation outside $\Omega$ or infinitely many operations from $\Lambda$ or $\Omega$, respectively.

The cost of computing $U(f)$, cost $(U, f)$, is given by

$$
\operatorname{cost}(U, f)=\operatorname{cost}(N, f)+\operatorname{cost}(\phi, N(f)) \text {. }
$$

Depending on the setting, the cost of $U$ is defined as follows. In the worst case setting

$$
\operatorname{cost}(U)=\sup _{f \in F} \operatorname{cost}(U, f),
$$

and in the average case setting

$$
\operatorname{cost}(U)=\int_{F} \operatorname{cost}(U, f) \mu(d f) .
$$

We are ready to define the basic notion of $\varepsilon$-complexity. The $\varepsilon$-complexity is defined as the minimal cost among all $U$ with error at most $\varepsilon$,

$$
\operatorname{comp}(\varepsilon)=\inf \{\operatorname{cost}(U): U \text { such that } e(U) \leq \varepsilon\} .
$$

(Here we use the convention that the infimum of the empty set is taken to be infinity.) Depending on the setting, this defines the worst case or average case $\varepsilon$-complexity.

We stress that we take the infimum over all possible $U$ for which the error does not exceed $\varepsilon$. Since $U$ can be identified with the pair $(N, \phi)$, where $N$ is the information and $\phi$ is the algorithm that uses that information, this means that we take the infimum over all information $N$ consisting of information operations from the class $\Lambda$, and over all algorithms $\phi$ that use $N$ such that $(N, \phi)$ computes approximations with error at most $\varepsilon$.

Remark. The complexity depends on the set $\Lambda$ of permissible information operations and on the set $\Omega$ of permissible combinatory operations. Both sets are necessary to define the complexity of a problem. This is beneficial because the dependence of complexity on $\Lambda$ and $\Omega$ enriches the theory; it enables us to study the power of specified information or combinatory operations. We illustrate the role of $\Lambda$ and $\Omega$ by a number of examples. 
We begin with the role of $\Lambda$. Assume that $F$ is a subset of a linear space of functions. Let $\Lambda_{1}$ consist of all linear functionals, and let $\Lambda_{2}$ consist of function evaluations. For many applications $\Lambda_{2}$ is more practical. Let $\Omega$ be defined as above.

Consider the integration example. For this problem, $\Lambda_{1}$ is not a reasonable choice since any integral could be computed exactly with cost $c$. For $\Lambda_{2}$, we get the multivariate integration problem discussed in this section.

Consider next the approximate solution of $2 m$ th-order elliptic linear partial differential equations whose right-hand side belongs to the unit ball of $H^{r}(D)$ for a bounded simply-connected $C^{\infty}$ region $D$ of $\mathbb{R}^{d}$. Let $G=H^{m}(D)$. Werschulz has shown that the worst case complexity in the class $\Lambda_{1}$ is proportional to $\varepsilon^{-d /(r+m)}$, and in the class $\Lambda_{2}$ it is proportional to $\varepsilon^{-d / r}$; a thorough study of this subject may be found in the research monograph Werschulz [91]. Thus, the complexity penalty for using $\Lambda_{2}$ rather than $\Lambda_{1}$ goes to infinity as $\varepsilon$ goes to zero for $m>0$; see also TWW [88, Chapter 5, Theorem 5.9]. On the other hand, Werschulz has shown that the complexity of Fredholm integral equations of the second kind is roughly the same for $\Lambda_{1}$ and $\Lambda_{2}$; see Werschulz [91] as well as TWW [88, Chapter $5, \S 6]$.

We now illustrate the role of $\Omega$ for the approximate solution of scalar complex polynomial equations of degree $d$ using complete information, i.e., $\Lambda$ consists of the identity mapping. Let $\Omega_{1}$ consist of the four arithmetic operations (over the complex field), and let $\Omega_{2}$ consist of the four arithmetic operations and complex conjugation. We confine ourselves to purely iterative algorithms. Then for $d \geq 4$, McMullen [85] proved that the problem cannot be solved for the class $\Omega_{1}$, whereas Shub and Smale [86] proved that the problem can be solved for the class $\Omega_{2}$. The positive result of Shub and Smale [86] also holds for systems of complex multivariate polynomials of degree $d$. Hence, the arithmetic operations are too weak for approximate polynomial zero finding, whereas also permitting complex conjugation supplies enough power to solve the problem.

Example (continued). For the integration problem, the model of computation states that one function evaluation costs $c$, and each arithmetic operation, comparisons of real numbers, and evaluations of certain elementary functions can be performed exactly at unit cost. Usually $c \gg 1$.

The worst case $\varepsilon$-complexity for the unit ball of $W_{p}^{r, d}$ is as follows. For $p r>d$,

$$
\operatorname{comp}(\varepsilon)=\Theta\left(c \varepsilon^{-d / r}\right) \quad \text { as } \varepsilon \rightarrow 0 ;
$$

see Novak [88] for a recent survey. Take $p=+\infty$. Then for $d$ large relative to $r$, the worst case $\varepsilon$-complexity is huge even for moderate $\varepsilon$. Furthermore, if only continuity of functions is assumed, then the problem cannot be solved since $\operatorname{comp}(\varepsilon)=+\infty$.

For the average case setting, let $F$ be the unit ball in the sup norm of continuous functions. Let $\mu$ be a truncated classical Wiener sheet measure; see, e.g., TWW [88, p. 218]. Then using results from number theory concerning discrepancy (see Roth $[54,80]$ ), we have

$$
\operatorname{comp}(\varepsilon)=\Theta\left(c \varepsilon^{-1}\left(\log \varepsilon^{-1}\right)^{(d-1) / 2}\right) \quad \text { as } \varepsilon \rightarrow 0 ;
$$

see $\mathrm{W}[87,91]$. Thus, the average case complexity depends only mildly on the 
dimension $d$. (The same $\Theta$ result holds if the unit ball is replaced by the entire space of continuous functions.) To get an approximation with cost proportional to $\operatorname{comp}(\varepsilon)$, it is enough to compute the arithmetic mean $n^{-1} \sum_{i=1}^{n} f\left(x_{i}\right)$, where $n=\Theta\left(\varepsilon^{-1}\left(\log \varepsilon^{-1}\right)^{(d-1) / 2}\right)$, and the points $x_{i}$ are derived from Hammersley points.

A goal of IBC is to find or estimate the $\varepsilon$-complexity, and to find an $\varepsilon$ complexity optimal $U$, or equivalently, an $\varepsilon$-complexity optimal pair $(N, \phi)$. By $\varepsilon$-complexity optimality of $U$ we mean that the error of $U$ is at most $\varepsilon$ and the cost of $U$ is equal to, or not much greater than, the $\varepsilon$-complexity. For a number of problems this goal has been achieved due to the work of many researchers.

Many computational problems can be formulated using the approach outlined above. For some problems, including the two matrix computation problems discussed in $\mathrm{P}$ [92], we need a more general formulation. We now briefly discuss this more general formulation; details can be found in TWW $[83,88]$.

Let $F$ and $G$ be given sets, and $W$ be a given mapping

$$
W: F \times[0,+\infty) \rightarrow 2^{G} .
$$

We assume that $W(f, 0)$ is nonempty and grows as $\varepsilon$ increases, i.e., for any $\varepsilon_{1} \leq \varepsilon_{2}$ we have $W\left(f, \varepsilon_{1}\right) \subset W\left(f, \varepsilon_{2}\right), \forall f \in F$.

We now wish to compute an element $U(f)$ which belongs to $W(f, \varepsilon)$ for all $f \in F$. The definitions of $U$ as well as the cost of $U$ are unchanged. The error of $U$ is now defined as follows. The error of $U$ for $f$ from $F$ is

$$
e(U, f)=\inf \{\eta: U(f) \in W(f, \eta)\} .
$$

Then the error of $U$ is defined as $e(U)=\sup _{f \in F} e(U, f)$ in the worst case setting, and $e(U)=\left(\int_{F} e^{2}(U, f) \mu(d f)\right)^{1 / 2}$ in the average case setting. Note that for

$$
W(f, \varepsilon)=\{g \in G:\|S(f)-g\| \leq \varepsilon\}
$$

we have $e(U, f)=\|S(f)-U(f)\|$ and the two formulations coincide.

Finally, we illustrate how the two matrix computation problems fit in this formulation.

(i) Large linear systems. We wish to approximate the solution of a large linear system $A z=b$ by computing a vector $x$ with residual at most $\varepsilon,\|A x-b\|$ $\leq \varepsilon$. Here, $b$ is a given vector, $\|b\|=1$, and $A$ belongs to a class $F$ of $n \times n$ nonsingular matrices. The vectors $x$ are computed by using matrixvector multiplications $A z$ for any vector $z$.

This problem corresponds to taking $G=\mathbb{R}^{n}$ and

$$
W(A, \varepsilon)=\{x \in G:\|A x-b\| \leq \varepsilon\}, \quad \forall A \in F .
$$

The class $\Lambda$ of information operations is now given by

$$
\begin{aligned}
\Lambda=\left\{L: F \rightarrow \mathbb{R}^{n}: \text { there exists a vector } z \in \mathbb{R}^{n}\right. \\
\text { such that } L(A)=A z, \forall A \in F\} .
\end{aligned}
$$

(ii) Eigenvalue problem. For a matrix $A$ from a class $F$ of $n \times n$ symmetric matrices, we wish to compute an approximate eigenpair $(x, \lambda)$, where $x \in \mathbb{R}^{n}$ with $\|x\|=1$, and $\lambda \in \mathbb{R}$, such that

$$
\|A x-\lambda x\| \leq \varepsilon\|A\| .
$$


As in (i), the pairs $(x, \lambda)$ are computed by using matrix-vector multiplications.

This problem corresponds to taking $G=B^{n} \times \mathbb{R}$, where $B^{n}$ is the unit sphere of $\mathbb{R}^{n}$, and

$$
W(A, \varepsilon)=\{(x, \lambda) \in G:\|A x-\lambda x\| \leq \varepsilon\|A\|\}, \quad \forall A \in F .
$$

The class $\Lambda$ is the same as in (i).

\section{THE ROLE OF INFORMATION}

Information is central to IBC. We indicate briefly why the distinction between information and algorithm is so powerful. We then respond to two general criticisms in $\mathrm{P}$ [92] regarding information.

As explained in $\S 2$, the approximation $U(f)$ is computed by combining information operations from the class $\Lambda$. Let $y=N(f)$ denote this computed information. In general, the operator $N$ is many-to-one, and therefore the set $N^{-1}(y)$ consists of many elements of $F$ that cannot be distinguished from $f$ using $N$. Then the set $S N^{-1}(y)$ consists of all elements from $G$ which are indistinguishable from $S(f)$. Since $U(f)$ is the same for any $f$ from the set $N^{-1}(y)$, the element $U(f)$ must serve as an approximation to any element $g$ from the set $S N^{-1}(y)$. It is clear that the quality of the approximation $U(f)$ depends on the "size" of the set $S N^{-1}(y)$. In the worst case setting, define the radius of information $r(N)$ as the maximal radius of the set $S N^{-1}(y)$ for $y \in N(F)$. (The radius of the set $A$ is the radius of the smallest ball which contains the set $A$.)

Clearly, the radius of information $r(N)$ is a sharp lower bound on the worst case error of any $U$. We can guarantee an $\varepsilon$-approximation iff $r(N)$ does not exceed $\varepsilon$ (modulo a technical assumption that the corresponding infimum is attained).

The cost of computing $N(f)$ is at least $c n$, where $c$ stands for the cost of one information operation, and $n$ denotes their number in the information $N$. By the $\varepsilon$-cardinality number $m(\varepsilon)$ we mean the minimal number $n$ of information operations for which the information $N$ has radius $r(N)$ at most equal to $\varepsilon$. From this we get a lower bound on the $\varepsilon$-complexity in the worst case setting,

$$
\operatorname{comp}(\varepsilon) \geq \operatorname{cm}(\varepsilon) .
$$

For some problems (see TWW [88, Chapter $5, \S 5.8]$ ) it turns out that it is possible to find an information operator $N_{\varepsilon}$ consisting of $m(\varepsilon)$ information operations, and a mapping $\phi_{\varepsilon}$ such that the approximation $U(f)=\phi_{\varepsilon}\left(N_{\varepsilon}(f)\right)$ has error at most $\varepsilon$ and $U(f)$ can be computed with cost at most $(c+2) m(\varepsilon)$. This yields an upper bound on the $\varepsilon$-complexity,

$$
\operatorname{comp}(\varepsilon) \leq(c+2) m(\varepsilon) .
$$

Since usually $c \gg 1$, the last two inequalities yield the almost exact value of the $\varepsilon$-complexity,

$$
\operatorname{comp}(\varepsilon) \simeq c m(\varepsilon) .
$$

This also shows that the pair $\left(N_{\varepsilon}, \phi_{\varepsilon}\right)$ is almost $\varepsilon$-complexity optimal.

In each setting of IBC one can define a radius of information such that we can guarantee an $\varepsilon$-approximation iff $r(N)$ does not exceed $\varepsilon$; see TWW [88]. This permits one to obtain complexity bounds in other settings. 
What is the essence of this approach? The point is that the radius of information as well as the $\varepsilon$-cardinality number $m(\varepsilon)$ and the information $N_{\varepsilon}$ do not depend on particular algorithms, and they can often be expressed entirely in terms of well-known mathematical concepts. Depending on the setting and on the particular problem, the radii of information, the $\varepsilon$-cardinality numbers, and the information $N_{\varepsilon}$ are related to Kolmogorov and Gelfand $n$-widths, $\varepsilon$ entropy, the traces of correlation operators of conditional measures, discrepancy theory, the minimal norm of splines, etc.

In summary, there are two reasons why one can sometimes obtain sharp bounds on $\varepsilon$-complexity in IBC. The first is the distinction between information and algorithm. The second is that, due to this distinction, one can draw on powerful results in pure and applied mathematics.

We now respond to two central criticisms in $P$ [92] regarding information. He asserts:

(i) The information is specified (or given) and therefore this "is not complexity theory;" see P [92, 2.A].

(ii) There is an "artificial distinction between information and algorithm;" see $P[92,1]$.

(i) P [92] repeatedly asserts that the information is "specified" or "given." We have already referred to this misconception in our introduction and will amplify our response here.

Varying the information and the algorithms is characteristic of IBC. (For problems for which information is complete, i.e., $N$ is one-to-one, only the algorithms can be varied.) The definition of computational complexity in our work always entails varying both information and algorithms; see, for example, TW [80, Chapter 1, Definition 3.2], TWW [83, Chapter 5, §3], W [85, 2.5], PW [87, II], TWW [88, Chapter $3, \S 3]$.

Furthermore the study of optimal information, which of course makes sense only if the information is being varied, is a constant theme in our work; see, for example, TW [80, Chapters 2 and 7], TWW [83, Chapter 4], W [85, 3.5], PW [87, III D, V C], TWW [88, Chapter 4, §5.3, Chapter 6, §5.5].

Here, we have responded to criticism (i) in general. In $\S \S 5$ and 6 we respond for the case of matrix computations.

(ii.1) $\mathbf{P}[92,1]$ claims there is an "artificial distinction between information and algorithm." That is, he argues that writing the approximation $U(f)=$ $\phi(N(f))$ is sometimes restrictive. We are surprised that he does not produce a single example to back his claim.

(ii.2) $\mathbf{P}$ [92, Abstract] states that "a sharp distinction is made between information and algorithms restricted to this information. Yet the information itself usually comes from an algorithm and so the distinction clouds the issues and can lead to true but misleading inferences."

We once again explain our view of the issues involved here using a simple integration example.

As in $\S 2$ assume that we can compute function values. How can we approximate the integral of $f$ ? The approximation $U(f)$ can be computed by evaluating $f$ at a number of points, say at $x_{1}, x_{2}, \ldots, x_{n}$, and then the computed values $f\left(x_{i}\right)$ are combined to get $U(f)$. Computations involving $f\left(x_{i}\right)$, the adaptive selection of the points $x_{i}$, and the adaptive choice of $n$ constitute 
the information $N(f)$. Denoting by $\phi$ the mapping which combines $N(f)$, we get $U(f)=\phi(N(f))$.

We do not understand why this is restrictive, why it clouds the issues, and why it leads to "true but misleading inferences." As explained in the first part of this section, the distinction between information and algorithm sometimes enables us to find sharp bounds on complexity.

\section{The Domain $F$}

A basic concept in IBC is the domain $F$. A central criticism of IBC in P [92] concerns $F$. The assertion is that there are two difficulties with $F$ :

(i) There is no need for $F$.

(ii) There should be a charge for knowing membership in $F$.

Concerning (i), the second "high level criticism" P [92, 2.B] states:

"The ingredient of IBCT that allows it to generate irrelevant results is the problem class $F$. $F$ does not appear in our brief description of the theory in the second paragraph of $\S 1$ because it is not a logically essential ingredient but rather a parameter within IBCT."

Concerning (ii), P [92, Abstract] states:

"By overlooking $F$ 's membership fee the theory sometimes distorts the economics of problem solving in a way reminiscent of agricultural subsidies."

First, why is $F$ needed?

(i.1) The set $F$ is necessary since it is the domain of the operator $S$, or part of the domain of the operator $W$.

One need not say anything further; an operator must have a domain. Nevertheless we will add a few additional points regarding the domain $F$.

(i.2) For discrete or finite-dimensional problems one can sometimes take the "maximal" set as $F$. Thus, in studying the complexity of matrix multiplications one usually takes $F$ as the set of all $n \times n$ matrices. In graph-theoretic complexity one often takes $F$ as the set of all graphs $(V, E)$, where $V$ is the set of vertices and $E$ is the set of edges.

However, for infinite-dimensional problems one cannot obtain meaningful complexity results if $F$ is too large. For example, the largest $F$ one might take for integration is the set of Lebesgue-integrable functions, but then $\operatorname{comp}(\varepsilon)=$ $+\infty, \forall \varepsilon \geq 0$ in the worst case setting. The $\varepsilon$-complexity remains infinite even if $F$ is the set of continuous functions.

To make the complexity of an infinite-dimensional problem finite, one must take a smaller $F$ in the worst case setting or switch to the average case setting. Thus, as we saw in $\S 2$, in the average case setting with a Wiener measure, the complexity is finite even if $F$ is the set of continuous functions.

(i.3) The use of $F$ is not confined to IBC. In discrete computational complexity researchers often use a set $F$ which is smaller than the maximal set. For example, if $F$ is the set of all graphs then many problems are NP-complete. If $F$ is a specified smaller set, then depending on the problem it may remain NP-complete or it may be solvable in polynomial time. See, for example, Garey and Johnson [79].

(i.4) We believe the dependence of complexity on $F$ is part of the richness of IBC. For example, in the integration problem it is interesting to know 
how complexity depends on the number of variables and the smoothness of the integrands.

(i.5) For a moment, we specialize our remarks to matrix computations. One could study the complexity of large linear systems for the set $F$ of all invertible matrices of order $n$. Then to compute an $\varepsilon$-approximation one would have to recover the matrix $A$ by computing $n$ matrix-vector multiplications; this is a negative result.

We find criticism (i) particularly odd since an entire book, Parlett [80], is devoted to only the eigenvalue problem for symmetric matrices. The reason is, of course, that the algorithms and the analysis for the symmetric eigenvalue problem are very different than for arbitrary matrices. But then why is the concept of $F$ so elusive?

Researchers in numerical linear algebra often consider other important subsets of matrices such as tridiagonal, Toeplitz, or Hessenberg matrices.

We turn to the criticism that there should be a charge for knowing membership in $F$.

(ii. 1) Is IBC being held to a higher standard? Do researchers in other disciplines charge for $F$ ? For example, researchers in numerical analysis often analyze the cost and error of important algorithms. The analysis depends on $F$. To give a simple example, the analysis of the composite trapezoidal rule usually requires that the second derivative of the integrand is bounded. There is no charge for membership in $F$. Indeed, how would one charge for knowing that a function has a bounded second derivative?

(ii.2) We believe that $\mathbf{P}[92]$ confuses two different problems:

(a) approximation of $S(f)$ for $f$ from $F$,

(b) the domain membership problem; that is, does $f$ belong to $F$ ?

Domain membership is an interesting problem which may be formulated within the IBC framework, although it has nothing to do with the original problem of approximating $S(f)$ for $f \in F$.

We outline how this may be done. First, to make the domain membership problem meaningful we must define the domain of $f$, say the set $\bar{F}$, in such a way that the logical values of $f \in F$ vary with $f$ from $\bar{F}$, i.e., $\varnothing \neq F \cap \bar{F} \neq \bar{F}$. Let $\bar{S}: \bar{F} \rightarrow\{0,1\} \subset \mathbb{R}$ be given by

$$
\bar{S}(f)=\chi_{F}(f), \quad \forall f \in \bar{F},
$$

where $\chi_{F}$ is the characteristic (indicator) function of $F$.

Then the problem is to compute $\bar{S}(f)$ exactly or approximately. Observe that we now assume that $f \in \bar{F}$ just as we assumed that $f \in F$ for problems of type (a).

For the domain membership problem we charge for computing an approximation to $\bar{S}(f)$, and the complexity of the domain membership problem is the minimal cost of verifying whether $f \in F$.

In the worst case setting, only the exact computation of $\bar{S}(f)$ makes sense since for $\varepsilon \geq \frac{1}{2}$ the problem is trivial, and for $\varepsilon<\frac{1}{2}$ it is the same as for $\varepsilon=0$. However for the average case or probabilistic settings, an $\varepsilon$-approximation may be reasonable. For instance we may wish to compute $\bar{S}(f)$ with probability $1-\varepsilon$.

It is easy to see that, in general, the domain membership problem cannot be 
solved in the worst case setting. To illustrate this, let $\bar{F}$ be the set of continous functions, and let $F$ be the set of $r$ times continuously differentiable functions, $r \geq 1$. Let the class $\Lambda$ of information operations consist of function values. It is obvious that knowing $n$ values of $f$, no matter how large $n$ may be, there is no way to verify whether $f$ is a member of $F$.

The domain membership problem can be studied in the average case or probabilistic settings. Its complexity may be large or small depending on $\bar{F}$ and $F$. An example of work for this problem is Gao and Wasilkowski [90] who study a particular domain membership problem.

(ii.3) Finally, we are at a loss to understand the following sentence from $\mathrm{P}$ [92, 2.B], "Whenever $F$ is very large (for example, the class of continuous functions or the class of invertible matrices) then it is realistic to assign no cost to it." Why is it realistic to assign no cost for "large" $F$, and why is it necessary to assign cost to "small" $F$ ? Where is the magic line which separates large $F$ from small $F$ ?

\section{LARGE LINEAR SYSTEMS}

We briefly describe IBC research on large linear systems and then respond to the criticisms in $\mathrm{P}$ [92]. Let

$$
A x=b,
$$

where $A \in F$, and $F$ is a class of $n \times n$ nonsingular matrices. Here $b$ is a known $n \times 1$ vector normalized such that $\|b\|=1$, and $\|\cdot\|$ stands for the spectral norm.

Our problem is defined as follows. For any $A \in F$ and any $\|b\|=1$ compute an $\varepsilon$-approximation $x$,

$$
\|A x-b\| \leq \varepsilon .
$$

Usually $A$ is sparse and therefore $A z$ can be computed in time and storage proportional to $n$. It is therefore reasonable for large linear systems to assume that the class $\Lambda$ of information operations consists of matrix-vector multiplications. That is, we can compute $A z_{1}, A z_{2}, \ldots, A z_{k}$, where $z_{i}$ may depend on the known vector $b$ and on the previously computed vectors $A z_{1}, \ldots, A z_{i-1}$. To stress that the right-hand side vector $b$ is known we slightly abuse the notation of $\S 2$ and denote

$$
N_{k}(A, b)=\left[b, A z_{1}, \ldots, A z_{k}\right], \quad A \in F,
$$

as the information about the problem. The number $k$ is called the cardinality of information. For this to be of interest, we need $k \ll n$.

Krylov information is the special case when we take $z_{1}=b$ and $z_{i}=A z_{i-1}$. Thus Krylov information is given by

$$
N_{k}^{\mathrm{Kr}}(A, b)=\left[b, A b, \ldots, A^{k} b\right] .
$$

In what follows we will use the concept of orthogonal invariance of the class $F$. The class $F$ is orthogonally invariant iff

$$
A \in F \text { implies } Q^{\mathrm{T}} A Q \in F
$$

for any orthogonal matrix $Q$, i.e., satisfying $Q^{\mathrm{T}} Q=I$.

Examples of orthogonally invariant classes include many of practical interest such as symmetric matrices, symmetric positive definite matrices, and matrices with uniformly bounded condition numbers. 
We first discuss optimal information for large linear systems which is defined as follows. The $\varepsilon$-cardinality number $m(\varepsilon)$ (see $\S 3$ ) denotes now the minimal cardinality $k$ of all information $N_{k}$ of the form (5.1) with $r\left(N_{k}\right) \leq \varepsilon$. Obviously, $m(\varepsilon)$ depends on the class $F$ and the class $\Lambda$. The information $N_{k}^{*}$ is optimal iff $k=m(\varepsilon)$ and $r\left(N_{k}^{*}\right) \leq \varepsilon$.

Remark. In $\S 2$ we define the $\varepsilon$-complexity optimality of a pair $(N, \phi)$. In this section optimality of information $N_{k}^{*}$ is introduced. How are these two optimality notions related?

In general, they are not. However, as already indicated in $\S 2$, for many problems the cost of computing $N_{k}^{*}(A, b)$ is proportional to $\operatorname{cm}(\varepsilon)$ and there exists an algorithm $\phi^{*}$ that uses $N_{k}^{*}$ and has error $\varepsilon$ and combinatory cost proportional to $m(\varepsilon)$. Then the pair $\left(N_{k}^{*}, \phi^{*}\right)$ is (almost) $\varepsilon$-complexity optimal. In this case, the two notions of optimality coincide and the complexity analysis reduces to the problem of finding optimal information. Details may be found in TWW [88, Chapter 4, §4].

In TW [84] we conjecture that for the class $\Lambda$ of matrix-vector multiplications and for any orthogonally invariant $F$, Krylov information is optimal.

Chou [87], based on Nemirovsky and Yudin [83], shows that Krylov information is optimal modulo a multiplicative factor of 2. More precisely, let $m^{\mathrm{Kr}}(\varepsilon)$ denote the minimal cardinality $k$ of Krylov information for which $r\left(N_{k}^{\mathrm{Kr}}\right) \leq \varepsilon$. For any orthogonally invariant class $F$, we have

$$
m(\varepsilon) \leq m^{\mathrm{Kr}}(\varepsilon) \leq 2 m(\varepsilon)+2 .
$$

Recently, Nemirovsky [91] shows that for a number of important orthogonally invariant classes $F$ and for $m(\varepsilon) \leq \frac{1}{2}(n-3)$, Krylov information is optimal,

$$
m(\varepsilon)=m^{\mathrm{Kr}}(\varepsilon) \text {. }
$$

We now discuss algorithms that use Krylov information. We recall the definition of the classical minimal residual (mr) algorithm; see, e.g., Stiefel [58]. The mr algorithm, $\phi^{\mathrm{mr}}$, uses Krylov information $N_{k}^{\mathrm{Kr}}(A, b)$ and computes the vector $x_{k}$ such that

$$
\begin{aligned}
\left\|A x_{k}-b\right\|=\min \left\{\left\|W_{k}(A) b\right\|:\right. & W_{k} \text { is a polynomial } \\
& \text { of degree } \left.\leq k \text { and } W_{k}(0)=1\right\} .
\end{aligned}
$$

Thus, by definition the $\mathrm{mr}$ algorithm minimizes the residual in the class of polynomial algorithms.

The $\mathrm{mr}$ algorithm has many good properties. Let $m^{\mathrm{Kr}}\left(\varepsilon, \phi^{\mathrm{mr}}\right)$ denote the minimal cardinality of Krylov information needed to compute an $\varepsilon$-approximation by the $\mathrm{mr}$ algorithm. Obviously, $m^{\mathrm{Kr}}(\varepsilon)$ denotes the minimal cardinality of Krylov information needed to compute an $\varepsilon$-approximation in the class of all algorithms. For any orthogonally invariant class $F$, we have (see TW [84])

$$
m^{\mathrm{Kr}}(\varepsilon) \leq m^{\mathrm{Kr}}\left(\varepsilon, \phi^{\mathrm{mr}}\right) \leq m^{\mathrm{Kr}}(\varepsilon)+1 .
$$

These bounds are sharp. That is, for some $F$ we have $m^{\mathrm{Kr}}(\varepsilon)=m^{\mathrm{Kr}}\left(\varepsilon, \phi^{\mathrm{mr}}\right)$, and for other $F$ we have $m^{\mathrm{Kr}}\left(\varepsilon, \phi^{\mathrm{mr}}\right)=m^{\mathrm{Kr}}(\varepsilon)+1$.

For all practically important cases, $m^{\mathrm{Kr}}(\varepsilon)$ is large and there is no significant difference between $m^{\mathrm{Kr}}\left(\varepsilon, \phi^{\mathrm{mr}}\right)$ and $m^{\mathrm{Kr}}(\varepsilon)$. Therefore the $\mathrm{mr}$ algorithm is always recommended as long as $F$ is orthogonally invariant. 
The mr algorithm minimizes, up to an additive term of 1 , the number of matrix-vector multiplications needed to compute an $\varepsilon$-approximation among all algorithms that use Krylov information in an orthogonally invariant class $F$. In this sense, the $\mathrm{mr}$ algorithm is Krylov-optimal, or for brevity, optimal.

We comment on the mr algorithm.

(1) The $\mathrm{mr}$ algorithm computes $x_{k}$ without using the additional properties of $A, A \in F$, given in the definition of the class $F$. This is desirable since the computation of $x_{k}$ is the same for all $F$. The vector $x_{k}$ can be computed by the well-known three-term recurrence formula using at most $10 k n$ arithmetic operations.

(2) Although the mr algorithm competes with all algorithms, in particular with algorithms that may use the additional properties of $A$ given in the definition of $F$, the $\mathrm{mr}$ algorithm can lose at most one insignificant step. Equivalently, one may say that for any orthogonally invariant class $F$, the a priori information about the class $F$ and the fact that $A \in F$ is worth at most one step.

(3) On the other hand, if $F$ is not orthogonally invariant then the $\mathrm{mr}$ algorithm may lose its good properties. Example 3.5 of TW [84] provides such a class for which the worst happens; the mr algorithm takes $n$ steps to solve the problem, whereas the optimal algorithm, which is nonpolynomial, takes only one step.

For an orthogonally invariant class $F$ and for the class $\Lambda$ of matrix-vector multiplications, these results yield that the pair Krylov information and $\mathrm{mr}$ algorithm is (almost) $\varepsilon$-complexity optimal in the sense of $\S 2$. Furthermore, we have rather tight bounds on the worst case complexity. More precisely,

$$
\operatorname{comp}(\varepsilon)=\operatorname{cam}^{\mathrm{Kr}}\left(\varepsilon, \phi^{\mathrm{mr}}\right),
$$

where $c$ is the cost of one matrix-vector multiplication and

$$
a \in\left[0.5-1 / m^{\mathrm{Kr}}\left(\varepsilon, \phi^{\mathrm{mr}}\right), 1+10 n / c\right] .
$$

For small $\varepsilon$ and $c \gg n$, we have roughly $a \in\left[\frac{1}{2}, 1\right]$.

Because of (5.2), the problem of obtaining the complexity reduces to the problem of finding $m^{\mathrm{Kr}}\left(\varepsilon, \phi^{\mathrm{mr}}\right)$. This number is known for some classes $F$; see TW [84] and TWW [88, Chapter 5, §9]. We discuss two classes:

$$
\begin{array}{ll}
F_{1}=\left\{A: A=A^{\mathrm{T}}>0,\right. & \text { and } \left.\|A\|_{2}\left\|A^{-1}\right\|_{2} \leq M\right\}, \\
F_{2}=\left\{A: A=A^{\mathrm{T}},\right. & \text { and } \left.\|A\|_{2}\left\|A^{-1}\right\|_{2} \leq M\right\} .
\end{array}
$$

That is, $F_{1}$ is the class of symmetric positive definite matrices with condition numbers bounded uniformly by $M$. Here $M$ is a given number, $M \geq 1$. The class $F_{2}$ differs from $F_{1}$ by the lack of positive definiteness.

For these two classes, the result of Nemirovsky [91] can be applied and for $m(\varepsilon) \leq \frac{1}{2}(n-3)$ we have better bounds on $a$; namely $a \in\left[1-1 / m^{\mathrm{Kr}}\left(\varepsilon, \phi^{\mathrm{mr}}\right)\right.$, $1+10 n / c]$. Thus, for small $\varepsilon$ and $c \gg n, a \simeq 1$.

For the class $F_{1}$, we have

$$
m^{\mathrm{Kr}}\left(\varepsilon, \phi^{\mathrm{mr}}\right)=\min \left\{n,\left\lceil\frac{\ln \left(\left(1+\left(1-\varepsilon^{2}\right)^{1 / 2}\right) / \varepsilon\right)}{\ln \left(\left(M^{1 / 2}+1\right) /\left(M^{1 / 2}-1\right)\right)}\right\rceil\right\}
$$


For small $\varepsilon$, large $M$, and $n>M^{1 / 2} \ln (2 / \varepsilon) / 2$, we have

$$
m^{\mathrm{Kr}}\left(\varepsilon, \phi^{\mathrm{mr}}\right) \simeq \frac{\sqrt{M}}{2} \ln \frac{2}{\varepsilon} .
$$

For the class $F_{2}$, we have

$$
m^{\mathrm{Kr}}\left(\varepsilon, \phi^{\mathrm{mr}}\right)=\min \left\{n, 2\left\lceil\frac{\ln \left(\left(1+\left(1-\varepsilon^{2}\right)^{1 / 2}\right) / \varepsilon\right)}{\ln ((M+1) /(M-1))}\right\rceil\right\} .
$$

For small $\varepsilon$, large $M$, and $n>M \ln (2 / \varepsilon)$, we have

$$
m^{\mathrm{Kr}}\left(\varepsilon, \phi^{\mathrm{mr}}\right) \simeq M \ln \frac{2}{\varepsilon} .
$$

These formulas enable us to compare the complexities for classes $F_{1}$ and $F_{2}$. For small $\varepsilon$, large $M$, and $n>2 M \ln (2 / \varepsilon)+3$, we have

$$
\frac{\operatorname{comp}\left(\varepsilon, F_{1}\right)}{\operatorname{comp}\left(\varepsilon, F_{2}\right)} \simeq \frac{1}{2 \sqrt{M}} \text {. }
$$

This shows how positive definiteness decreases the $\varepsilon$-complexity.

$P$ [92] has four "high level" criticisms of IBC research on the large linear systems problem. We also select three additional criticisms from $P$ [92, 4]. We shall respond to these seven criticisms. P [92] contains other misunderstandings and errors regarding this topic but we will not try the reader's patience by responding to each of these. We list the seven criticisms of $P$ [92]:

(i) IBC "is not complexity theory" since "the stubborn fact remains that restricting information to Krylov information is not part of the linear equations problem" $P[92,2 . \mathrm{A}]$.

(ii) "The trouble with this apparent novelty is that it is not possible to evaluate the residual norm $\|A z-b\|$ for those external $z$ because there is no known matrix $A$ (only Krylov information). So how can an algorithm that produces $z$ verify whether or not it has achieved its goal of making $\|A z-b\|<\varepsilon\|b\| " \mathrm{P}[92,2 . \mathrm{C}]$.

(iii) "The ingredient of IBCT that allows it to generate irrelevant results is the problem class $F$ [see paragraph 2 in (A)]. $F$ did not appear in our brief description of the theory in the second paragraph of $\S 1$ because it is not a logically essential ingredient but rather a parameter within IBCT;" P [92, 2.B].

(iv) "IBCT's suggestion that it goes beyond the well-known polynomial class of algorithms is more apparent than real;" $P[92,2 . C]$.

(v) "Here is a result of ours that shows why the nonpolynomial algorithms are of no interest in worst case complexity;" $\mathrm{P}[92,4.3]$.

(vi) "With a realistic class such as SPD (sym, pos. def.) MR is optimal (strongly) as it was designed to be, and as is well known;" $P[92,4.4]$.

(vii) "The theory claims to compare algorithms restricted solely to information $N_{j}$. So how could the Cheb algorithm obtain the crucial parameter $\rho$ ?;” P $[92,4.4]$.

We respond to each of these seven criticisms.

(i) IBC does not restrict information to Krylov information. The optimality of Krylov information in the class of matrix-vector multiplications is a conclusion, not an assumption. 
IBC does assume a class $\Lambda$ of information operations. The reasons why this is both necessary and beneficial were discussed in $\S 2$. Here we confine ourselves to certain classes relevant to large linear systems.

Let $\Lambda_{1}$ denote the class of matrix-vector multiplications. Then as described above, for an orthogonally invariant class $F$ we may conclude that Krylov information is optimal to within a multiplicative factor of at most 2. Furthemore, we may conclude that Krylov information and the $\mathrm{mr}$ algorithm are almost $\varepsilon$ complexity optimal. Rather tight bounds have been obtained on the complexity of important classes such as $F_{1}$ and $F_{2}$, see above. Additional classes of matrices are studied in TW [84].

Let $\Lambda_{2}$ denote the class of information operations where inner products of rows (or columns) of $A$ and an arbitrary vector $z$ can be computed. Rabin [72] studied the class $\Lambda_{2}$ for the exact solution of linear systems, $\varepsilon=0$, and for an arbitrary nonsingular matrix $A$. He proved that, roughly, $\frac{1}{2} n^{2}$ inner products are sufficient to solve the problem. No results are known for $\varepsilon>0$.

Let $\Lambda_{3}$ denote the class of information operations consisting of arbitrary linear functionals. Optimality questions for the class $\Lambda_{3}$ are posed in TW [84]. No results are known and we believe this to be a difficult problem.

Let $\Lambda_{4}$ denote the class of information operations consisting of continuous nonlinear functionals, and let $\Lambda_{5}$ denote the class of nonlinear functionals. In general, complexity results in $\Lambda_{4}$ and $\Lambda_{5}$ can be different; see Kacewicz and Wasilkowski [86] and Mathé [90]. For linear systems, these classes are too powerful since all entries of the matrix $A$ can be recovered by knowing the value of one continuous nonlinear functional. Thus, the $\varepsilon$-cardinality number is 1 even for $\varepsilon=0$; see TW [80, Chapter $7, \S 3$ ] for related material.

(ii) If the class $\Lambda$ consists of matrix-vector multiplications then, of course, we can evaluate the residual $\|A z-b\|$ for any $z$. If $z$ is outside of a Krylov subspace this requires one additional matrix-vector multiplication.

On the other hand, it is sometimes possible to guarantee that $\|A z-b\| \leq \varepsilon$, without computing the residual $\|A z-b\|$. This can be done by using a priori information that $A \in F$ and the computed Krylov information. An example of such a situation is provided by the Chebyshev algorithm for the class $F=$ $\left\{A=I-B: B=B^{\mathrm{T}},\|B\| \leq \rho<1\right\}$.

In general, if the assumptions are satisfied, IBC is predictive. The results of the theory guarantee an $\varepsilon$-approximation. One simply does the amount of work specified by the upper bound on the complexity. For important classes of matrices we have seen above that there are rather tight bounds on the complexity. Therefore this strategy does not require much more work than necessary.

For most problems there is no residual that can be checked. There are residuals for problems related to solving linear or nonlinear equations. In the multivariate integration example of $\S 2$, there is no residual that can be computed. Yet, IBC guarantees an $\varepsilon$-approximation by using a priori information about the class $F$.

(iii) We responded in general to the criticism that $F$ is not needed in $\S 4$; here we focus on large linear systems. On this problem $P[92,2 . B]$ states that "IBCT seems to use $F$ as a tuning parameter designed to keep $k<n$."

The domain $F$ is not a tuning parameter; it is needed for the problem to be well defined. The domain $F$ contains all a priori knowledge about matrices 
$A$. The more we know a priori, the smaller the domain $F$ becomes, and as $F$ becomes smaller, the problem becomes easier. Furthermore, a priori information is often available in practice. For example, matrices which occur in the approximation of elliptic partial differential operators are symmetric positive definite, often with known bounds on condition numbers.

Fortunately, many important classes which occur in practice are orthogonally invariant and the $\varepsilon$-complexity optimality of Krylov information and the $\mathrm{mr}$ algorithm may be applied.

Of course, numerical analysts use different algorithms for different classes of matrices (symmetric, positive definite, tridiagonal, Toeplitz, etc.) It is therefore all the more surprising that $\mathrm{P}$ [92] objects to the concept of the class $F$.

(iv) $\mathrm{P}[92,2 \mathrm{C}]$ claims that there is no need to go "beyond the well-known polynomial class of algorithms." It should be obvious that all algorithms must be allowed to compete if we want to establish lower bounds on complexity.

For orthogonally invariant classes it turns out that the restriction to the polynomial class of algorithms does not cause any harm since the classical mr algorithm may lose at most one insignificant step. But this had to be proven!

In fact, it is not uncommon in computational complexity that the known algorithms (that use the specific information) turn out to be optimal or close to optimal. Examples include the Horner algorithm for evaluating a polynomial, the finite element method with appropriate parameters for elliptic partial differential equations, or the bisection algorithm for approximating a zero of a continuous function that changes sign at the interval endpoints.

For large linear systems, a sufficient condition for almost $\varepsilon$-complexity optimality of Krylov information and the $\mathrm{mr}$ algorithm is orthogonal invariance of the class $F$. As mentioned above, Example 3.5 of TW [84] shows that if $F$ is not orthogonally invariant, the $\mathrm{mr}$ algorithm may lose its optimality. In this example the restriction to the polynomial class of algorithms is harmful because the optimal algorithm is nonpolynomial.

(v) $\mathrm{P}[92,4.3]$ supports his claim that nonpolynomial algorithms are not interesting by the Theorem of $\S 4.3$. This theorem holds for the class of SPD of all $n \times n$ symmetric positive definite matrices. In this theorem it is shown that for every nonpolynomial algorithm which computes an approximation outside the Krylov subspace for $A \in \mathrm{SPD}$, there exists a matrix from SPD which has the identical Krylov information as $A$ and for which the residual is arbitrarily large.

We do not understand why the Theorem of $\S 4.3$ and the one page sketch of its proof were supplied. The same statement can be found in Example 3.4 of TW [84]. In addition, Example 3.4 shows that polynomial algorithms are also not good for the class SPD; that is, $n$ matrix-vector multiplications are needed to compute an $\varepsilon$-approximation. The reason neither polynomial nor nonpolynomial algorithms are good is that the class SPD is too large.

We stress that Example 3.4 and the Theorem of $\S 4.3$ hold for $F=$ SPD. As mentioned above, for any orthogonally invariant class $F$ the nonpolynomial algorithms are not of interest since it has been proven that the $\mathrm{mr}$ algorithm is optimal, possibly modulo one matrix-vector multiplication. Also, as mentioned above, if $F$ is not orthogonally invariant, a nonpolynomial algorithm may be optimal.

(vi) P [92] claims that the mr algorithm is optimal "as it was designed to be" 
for the class SPD. This is simply not true. The mr algorithm is defined to be optimal in the class of polynomial algorithms. Optimality of the mr algorithm in the class of all algorithms for the class SPD requires a proof.

(vii) As already explained, the information that $A \in F=\{A=I-B: B=$ $\left.B^{\mathrm{T}},\|B\| \leq \rho<1\right\}$ is not used by the $\mathrm{mr}$ algorithm. This means that the $\mathrm{mr}$ algorithm does not use the parameter $\rho$ which is assumed known a priori and may be used by competing algorithms. The parameter $\rho$ is used by the Chebyshev algorithm and that is why the mr algorithm loses one step for the class $F . \mathrm{P}[92,4.4]$ turns the positive optimality result for the $\mathrm{mr}$ algorithm into the irrelevant question "how could the Chebyshev algorithm obtain the crucial parameter $\rho$ ?" By the way, the parameter $\rho$ is not so crucial if it decreases the number of steps by only one!

\section{LARGE EIGENVALUE PROBLEM}

$P$ [92] has three "high level" criticisms of the IBC research on the large eigenpair problem. He also criticizes the numerical testing. We shall respond to these four criticisms.

We list the four criticisms of $P$ [92]:

(i) Kuczyński [86] computes an unspecified eigenvalue; $P$ [92, 2.D].

(ii) IBC "is not complexity theory." The reason given is that "the stubborn fact remains that restricting information to Krylov information is not part ... of the eigenvalue problem;" $P[92,2 . A]$.

(iii) "The fact that $b$ is treated as prescribed data is quite difficult to spot;" $P[92,2 . E]$.

(iv) "The author has worked exclusively with tridiagonal matrices and has forgotten that the goal of the Lanczos recurrence is to produce a tridiagonal matrix! Given such a matrix one has no need of either Lanczos or GMR;" P [92, 5.5].

We respond to each of these four criticisms.

(i) P [92] is certainly correct in asserting that when only one or a few eigenvalues of a symmetric matrix are sought, then one typically desires a preassigned eigenvalue or a few preassigned eigenvalues. To be specific, assume that the largest eigenvalue is to be approximated.

It would be desirable to always guarantee that the largest eigenvalue $\lambda_{1}(A)$ of a large symmetric matrix $A$ can be computed to within error $\varepsilon$. Unfortunately, this cannot be done with less than $n$ matrix-vector multiplications, that is, without recovering the matrix $A$; see TWW [88, Chapter $5, \S 10]$. More precisely, let $F$ denote the class of all $n \times n$ symmetric matrices and let $\Lambda$ consist of matrix-vector multiplications. That is, $N(A)=\left[A z_{1}, \ldots, A z_{k}\right]$, where $z_{1}$ is an arbitrary vector and $z_{i}$ for $i \geq 2$ may depend arbitrarily on $A z_{1}, \ldots, A z_{i-1}$. Then for $k \leq n-1$, there exists no such $N$ and no algorithm $\phi$ which uses $N$ such that $U(A)=\phi(N(A))$ satisfies

$$
\left|\lambda_{1}(A)-U(A)\right| \leq \varepsilon\|A\|, \quad \forall A \in F .
$$

We are surprised that although TWW [88] is cited in P [92], he does not seem to be aware of this result.

Thus, the goal of computing an $\varepsilon$-approximation to the largest eigenvalue of a large symmetric matrix cannot be achieved, if less than $n$ matrix-vector 
multiplications are used. This is, of course, a worst case result. There are a number of options for coping with this negative result. One could stay with the worst case setting but settle for an unspecified eigenvalue. Or one could give up on the worst case guarantee and settle for a weaker one. We consider these options in turn.

(i.1) One option is to settle for an unspecified eigenvalue. More precisely, the problem studied by Kuczyński [86] and Chou [87] is defined as follows. For $A \in F$, compute $(x, \lambda)$ with $x \in \mathbb{R}^{n},\|x\|=1$, and $\lambda \in \mathbb{R}$, such that

$$
\|A x-\lambda x\| \leq \varepsilon\|A\| .
$$

Chou proved, modulo a multiplicative factor of 2, optimality of Krylov information $N(A)=\left[A b, \ldots, A^{k} b\right]$, where $b$ is a nonzero vector. Optimality of Krylov information holds independently of the choice of the vector $b$. Kuczyński proved, modulo an additive term of 2, optimality of the generalized minimal residual (gmr) algorithm that uses Krylov information. (Optimality of Krylov information and the gmr algorithm is understood as in $\S 5$. These optimality results hold for any orthogonally invariant class of matrices. )

Since the gmr algorithm has small combinatory cost, we conclude that the pair Krylov information and gmr algorithm is (almost) $\varepsilon$-complexity optimal. Kuczyński found good bounds on the worst case error of the gmr algorithm. Hence, for $n>\varepsilon^{-1}$, the worst case $\varepsilon$-complexity is given by

$$
\operatorname{comp}(\varepsilon)=\frac{a c}{\varepsilon},
$$

where $a$ roughly belongs to $\left[\frac{1}{4}, 1\right]$ and, as before, $c$ is the cost of one matrixvector multiplication.

(i.2) A second option is to attempt to approximate the largest eigenvalue but to settle for a weaker guarantee. $\mathrm{KW}[89]^{4}$ study this problem in the randomized setting. (See, e.g., TWW [88, Chapter 11] for a general discussion of the randomized setting.)

In particular, the Lanczos algorithm is studied. The Lanczos algorithm uses Krylov information $N(A)=\left[A b, A^{2} b, \ldots, A^{k} b\right]$ with a random vector $b$ which is uniformly distributed over the unit sphere of $\mathbb{R}^{n}$. The error is defined for a fixed matrix $A$ while taking the average with respect to the vectors $b$.

To date only an upper bound on the error of the Lanczos algorithm with randomized Krylov information has been obtained. This upper bound is proportional to $((\ln n) / k)^{2}$.

As always, to obtain complexity results both the information and the algorithm must be varied. Lower bounds are of particular interest. The complexity of approximating the largest eigenvalue in the randomized setting is open.

(ii) $\mathrm{P}[92,2 . \mathrm{A}]$ states “ $\ldots$ the stubborn fact remains that restricting information to Krylov information is not part ... of the eigenvalue problem."

Although we have mentioned several times in this paper that $\mathbf{P}$ [92] seems unaware of the results regarding optimality of Krylov information we are particularly surprised that he appears unaware of this result in the context of the large eigenvalue problem. P [92] repeatedly cites Kuczyński [86] where Chou's result is reported.

\footnotetext{
${ }^{4}$ This paper is mistakenly referred in $\mathrm{P}[92]$ as [ $\operatorname{Tr} \&$ Wo, 1990].
} 
(iii) $\mathbf{P}[92,2 . \mathrm{E}]$ states "the fact that $b$ is treated as prescribed data is quite difficult to spot." Perhaps the reason it is difficult to spot is that it is not prescribed.

What is assumed known? It is known a priori that $A$ is a symmetric $n \times n$ matrix. Furthermore, we are permitted to compute $A z_{1}, \ldots, A z_{k}$, where $z_{i}$ may be adaptively chosen. We are permitted to choose $z_{1}$, which is called $b$, arbitrarily. In choosing $b$ we cannot assume that $A$ is known, since the raison d'etre of methods for solving large eigenvalue problems is just that $A$ need not be known.

By the result quoted in (i), it is impossible to guarantee that we can find a vector $b$ such that an $\varepsilon$-approximation to the largest eigenvalue can be computed for all symmetric $n \times n$ matrices with $k<n$.

If Krylov information $A b, A^{2} b, \ldots, A^{k} b$ is used then the situation is even worse. Even for arbitrary $k$, i.e., even for $k \geq n$, an $\varepsilon$-approximation cannot be computed. Indeed, suppose we choose a vector $b$ and a matrix $A$ such that $A b=b$. Then Krylov information is reduced just to the vector $b$. The largest eigenvalue cannot be recovered (unless $n=1$ ). Thus, for any vector $b$ there are symmetric matrices $A$ for which Krylov information will not work.

Of course, one can choose $b$ randomly, as was discussed above. The average behavior with respect to vectors $b$ is satisfactory for all symmetric matrices. But then one is settling for a weaker guarantee of solving the problem.

$P$ [92, 2.E] claims that for Krylov information "satisfactory starting vectors are easy to obtain." This remark seems to confuse the worst case and randomized settings. To get a satisfactory starting vector $b$ in the worst case setting, the vector $b$ must be chosen using some additional information about the matrix $A$. If such information is not available, it is impossible to guarantee satisfactory starting vectors. On the other hand, in the randomized setting it is indeed easy to get satisfactory starting vectors.

(iv) $\mathbf{P}[92,5.5]$ complains that Kuczyński [86] tests only tridiagonal matrices.

There is no loss of generality in restricting the convergence tests of the Lanczos or gmr algorithms to tridiagonal matrices. That was done in Kuczyński [86] to speed up his tests. What is claimed in Kuczyński [86] for the pairs $(T R I, b), T R I$ a tridiagonal matrix and $b=e_{1}=[1,0, \ldots, 0]^{\mathrm{T}}$, is also true for the pairs $\left(Q^{\mathrm{T}} T R I Q, Q^{\mathrm{T}} b\right)$ for any orthogonal matrix $Q$. Obviously, the matrix $Q^{\mathrm{T}} T R I Q$ is not, in general, tridiagonal.

The confusion between the worst case and randomized settings is also apparent when $P$ [92] discusses numerical tests performed by Kuczyński [86] and by him.

For the unspecified eigenvalue problem, Kuczyński [86] compares the gmr and Lanczos algorithms in the worst case setting. These two algorithms cost essentially the same per step, and the gmr algorithm never requires more steps than the Lanczos algorithm. For some matrices, the gmr algorithm uses substantially fewer steps than the Lanczos algorithm. That is why in the worst case setting the gmr algorithm is preferable.

$P$ [92] performed his numerical tests for the Lanczos algorithm with random starting vectors $b$. Thus, he uses a different setting. It is meaningless to compare numerical results in different settings.

Finally, extensive numerical testing is also reported in $\mathrm{KW}$ [89] for approxi- 
mating the largest eigenvalue by the Lanczos algorithm with randomized starting vectors. The Lanczos algorithm worked quite well for all matrices tested. The numerical tests reported by $\mathrm{P}$ [92] and KW [89] show the efficiency of the Lanczos algorithm in the randomized setting.

\section{REFINEMENTS OF IBC}

Our response to the criticism in $P$ [92] does not mean that the current model assumptions of IBC are the only ones possible. On the contrary, we believe that in some circumstances these assumptions should be refined to improve the modelling of computational problems. We have mentioned the desirability of such refinements in, e.g., TWW [88, Chapter 3, §2.3] and W [85, §9]. In this section we will very briefly indicate some of the possible refinements and extensions of IBC, and indicate partial progress. This is preparatory to responding to several comments in $P$ [92].

Refinements and extensions of IBC include the following:

(1) We usually assume the real number model in a sequential model of computation where the cost of a combinatory operation is independent of the precision of the operands or of the result. Also of interest is a model where the cost of a combinatory operation depends on the precision (bit model) and/or on the particular operation. Parallel and distributed models of computation should also be studied. For examples of work in these directions see Bojańczyk [84] who studies the approximate solution of linear systems using a variable precision parallel model of computation, and Kacewicz [90] who studies initial value problems for both sequential and parallel models of computation.

(2) We usually assume that for every information operation $L \in \Lambda$ and for every $f \in F$ the computation of $L(f)$ costs $c, c>0$. Also of interest is a model where the cost of an information operation depends on $L, f$, and precision. For an example, see Kacewicz and Plaskota [90] who study linear problems in a model where the cost of information operations varies with the computed precision.

(3) Let $S$ be a linear operator. Then we often assume that the set $F$ is balanced and convex; TWW [88, Chapter $4, \S 5]$. In particular, for functions spaces, we often assume that $F$ is a Sobolev space of smoothness $r$ with a uniform bound on $\left\|f^{(r)}\right\|$. It is of interest to study $F$ which do not have such a nice structure.

$P[92,1]$ states "a handful of reservations about IBC have appeared in print." These "reservations" turn out to concern refinements of IBC. P [92] writes that Babuška [87] calls for realistic models. For example, Babuška points out that for some problems arising in practice the set $F$ does not consist of smooth functions but rather of functions which are piecewise smooth with singularities at unknown points. We agree that this is an important problem. A promising start has been made by Wasilkowski and Gao [89] on estimating a singularity of a piecewise smooth function in a probabilistic setting.

Babuška observes that the user may not know the class $F$ or not know $F$ exactly, and suggests the importance of algorithms which enjoy optimality properties for a number of classes. We agree that this is an important concern and a good direction for future research. See W $[85, \S 9.3]$ where this problem is 
called the "fat" $F$ problem and where partial results are discussed. One attack on this problem is to address the domain membership problem defined in $\S 4$. As indicated there, this can only be done with a stochastic assurance.

$P[92,1]$ asserts that in a review of TWW [83], Shub [87] "gives a couple of instances of unnatural measures of cost." (These words are from P [92], not from Shub [87].) Shub, in a generally favorable review (the reader may want to verify this), suggests circumstances when the cost of an information operation should vary. We concur.

\section{SUMMARY}

P $[92,2]$ states five high level criticisms of IBC. We responded to them in the following sections:

$\begin{array}{cc}\text { Criticism } & \text { Response } \\ \text { A } & 1,3,5,6 \\ \text { B } & 4,5,6 \\ \text { C } & 5 \\ \text { D } & 6 \\ \text { E } & 6\end{array}$

There are additional criticisms, and in $\S \S 5$ and 6 we responded to the ones which seem most important.

$P[92,1]$ states that "a handful of reservations about IBCT have appeared in print." He neglects mentioning the many favorable reviews. He cites two examples of reservations. We discussed the comments of Babuška [87] and Shub [87] in $\S 7$.

$P$ [92] is based upon the following syllogism:

(1) Major Premise: If two specific papers of IBC are misleading, then IBC is flawed.

(2) Minor Premise: Two specific papers of IBC regarding matrix computations are misleading.

(3) Conclusion: IBC is flawed.

We have shown that his reasons for believing the minor premise are mistaken.

\section{REFERENCES}

I. Babuška, Information-based numerical practice, J. Complexity (1987), 331-346.

N. S. Bakhvalov, On approximate calculation of integrals, Vestnik Moskov. Gos. Univ. Ser. Mat. Mekh. Astronom. Fiz. Khim. 4 (1959), 3-18. (Russian)

, On optimal bounds for the convergence of quadrature formulas and Monte-Carlo type integration methods for classes of functions, Numerical Methods for the Solution of Differential and Integral Equations and Quadrature Formulas, Nauka, Moscow, 1964, pp. 5-63. (Russian)

_ On the optimality of linear methods for operator approximation in convex classes of functions, U.S.S.R Comput. Math. and Math. Phys. 11 (1971), 244-249. (Russian)

L. Blum, M. Shub, and S. Smale, On a theory of computation and complexity over the real numbers: NP-completeness, recursive functions and universal machines, Bull. Amer. Math. Soc. (N.S.) 21 (1989), 1-46.

A. Bojańczyk, Complexity of solving linear systems in different models of computation, SIAM J. Comput. 21 (1984), 591-603.

A. W. Chou, On the optimality of Krylov information, J. Complexity 3 (1987), 26-40. 
F. Gao and G. W. Wasilkowski, On detecting regularity of functions, work in progress, 1990.

R. M. Garey and D. S. Johnson, Computers and intractability: A guide to the theory of NP-completeness, Freeman, New York, 1979.

M. Golomb and H. F. Weinberger, Optimal approximation and error bounds, On Numerical Approximation (R. E. Langer, ed.), Univ. of Wisconsin Press, Madison, WI, 1959, pp. 117-190.

B. Z. Kacewicz, On sequential and parallel solution of initial value problems, J. Complexity 6 (1990), 136-148.

B. Z. Kacewicz and L. Plaskota, On the minimal cost of approximating linear problems based on information with deterministic noise, Numer. Funct. Anal. Optim. (1990) (to appear).

B. Z. Kacewicz and G. W. Wasilkowski, How powerful is continuous nonlinear information for linear problems?, J. Complexity 2 (1986), 306-316.

J. Kiefer, Sequential minimax search for a maximum, Proc. Amer. Math. Soc. 4 (1953), 502-505.

J. Kuczyński, On the optimal solution of large eigenpair problems, J. Complexity 2 (1986), 131-162.

J. Kuczyński and $\mathrm{H}$. Woźniakowski, Estimating the largest eigenvalue by the power and Lanczos algorithms with a random start, Report, Dept. of Computer Science, Columbia University, 1989 (to appear in SIMAX).

P. Mathé, s-numbers in information-based complexity, J. Complexity 6 (1990), 41-66.

C. McMullen, Families of rational maps and iterative root-finding algorithms, Ph.D. thesis, Harvard University, Cambridge., MA, 1985.

A. S. Nemirovsky, On optimality of Krylov's information when solving linear operator equations, $\mathrm{J}$. Complexity 7 (1991), 121-130.

A. S. Nemirovsky and D. B. Yudin, Problem complexity and method efficiency in optimization, Wiley-Interscience, New York, 1983.

S. M. Nikolskij, On the problem of approximation estimate by quadrature formulas, Uspekhi. Mat. Nauk 5 (1950), 165-177. (Russian)

E. Novak, Deterministic and stochastic error bounds in numerical analysis, Lectures Notes in Math., vol. 1349, Springer-Verlag, Berlin, 1988.

E. W. Packel and J. F. Traub, Information-based complexity, Nature 328 (1987), 29-33.

E. W. Packel and H. Woźniakowski, Recent developments in information-based complexity, Bull. Amer. Math. Soc. (N.S.) 17 (1987), 9-36.

B. N. Parlett, The symmetric eigenvalue problem, Prentice-Hall, Englewood Cliffs, NJ, 1980. , Some basic information on information-based complexity theory, Bull. Amer. Math. Soc. (N.S.) 26 (1992), 3-27.

M. O. Rabin, Solving linear equations by means of scalar products, Complexity of Computer Computations (R. E. Miller and J. W. Thatcher, eds.), Plenum Press, New York, 1972, pp. 11-20.

K. F. Roth, On irregularities of distribution, Mathematika 1 (1954), 73-79. , On irregularities of distribution. IV, Acta Arith. 37 (1980), 67-75.

A. Sard, Best approximate integration formulas; best approximation formulas, Amer. J. Math. 71 (1949), 80-91.

M. Shub, Review of "Information, uncertainty, complexity"by J. F. Traub, G. W. Wasilkowski, and H. Woźniakowski (Addison-Wesley, Reading, MA, 1983), SIAM Re. 29 (1987), 495-496.

M. Shub and S. Smale, On the existence of generally convergent algorithms, J. Complexity 2 (1986), $2-11$.

I. J. Schoenberg, Spline interpolation and best quadrature formulas, Bull. Amer. Math. Soc. 70 (1964), 143-148.

E. Stiefel, Kernel polynomials in linear algebra and their numerical applications, NBS Appl. Math. 43 (1958), 1-22.

J. F. Traub, G. W. Wasilkowski, and H. Woźniakowski, Information, uncertainty, complexity, Addison-Wesley, Reading, MA, 1983.

Information-based complexity, Academic Press, New York, 1988.

J. F. Traub and H. Woźniakowski, A general theory of optimal algorithms, Academic Press, New York, 1980. 
On the optimal solution of large linear systems, J. Assoc. Comput. Mach. 31 (1984), 545-559.

, Information-based complexity: New questions for mathematicians, Math. Intelligencer 13 (1981), 34-43.

G. W. Wasilkowski and F. Gao, On the power of adaptive information for functions with singularities, Math. Comp. 58 (1992), pp. 285-304.

A. G. Werschulz, The computational complexity of differential and Integral equations, Oxford Univ. Press, Oxford, 1991.

H. Woźniakowski, A survey of information based-complexity, J. Complexity 1 (1985), 11-44.

_, Average complexity for linear operators over bounded domains, J. Complexity 3 (1987), 57-80.

Average case complexity of multivariate integration, Bull. Amer. Math. Soc. (N.S.) 24 (1991), 185-194.

(J. F. Traub) Department of Computer Science, Columbia University, New York, New YORK 10027

(H. Woźniakowski) Department of Computer Science, Columbia University and Institute of Informatics, University of Warsaw, Warsaw, Poland 